\title{
Internet Nameserver IPv4 and IPv6 Address Relationships
}

\author{
Arthur Berger \\ MIT CSAIL / Akamai \\ awberger@csail.mit.edu
}

\author{
Nicholas Weaver \\ ICSI / UCSD \\ nweaver@icsi.berkeley.edu
}

\author{
Robert Beverly \\ Naval Postgraduate School \\ rbeverly@nps.edu
}

\author{
Larry Campbell \\ Akamai \\ lcampbel@akamai.com
}

\begin{abstract}
The modern Domain Name System (DNS) provides not only resolution, but also enables intelligent client routing, e.g. for Content Distribution Networks (CDNs). The adoption of IPv6 presents CDNs the opportunity to utilize different paths when optimizing traffic, and the challenge of appropriately mapping IPv6 DNS queries. This work seeks to discover the associations between Internet DNS client resolver IPv6 address(es) and IPv4 address(es). We design and implement two new techniques, one passive and one active, to gather resolver pairings. The passive technique, deployed in Akamai's production DNS infrastructure, opportunistically discovered $674 \mathrm{k}$ (IPv4, IPv6) associated address pairs within a six-month period. We find that $34 \%$ of addresses are oneto-one, i.e. appear in no other pair, a fraction that increases to $\approx 50 \%$ when aggregating IPv6 addresses into /64 prefixes. The one-to-one associations are suggestive, but not a sufficient condition, of dual-stack DNS recursive resolvers. We further substantiate our inferences via PTR records and software versions, and manual verification of sample pairings by three major Network Operators. Complex associations, where e.g. distributed DNS resolution leads to inferred address groupings that span continents and many autonomous systems exist, a subset of which we explore in more depth using the active probing technique. Among potential uses, Akamai is currently utilizing screened output from the passive technique, in conjunction with prior knowledge of $\mathrm{IPv}_{\mathrm{v}}$, to inform IPv6 geolocation within its CDN.
\end{abstract}

\section{Categories and Subject Descriptors}

C.2.3 [Computer Communication Networks]: Network Operations-network monitoring

\section{General Terms}

Measurement, Experimentation

(c) 2013 Association for Computing Machinery. ACM acknowledges that this contribution was authored or co-authored by an employee, contractor or affiliate of the United States government. As such, the United States Government retains a nonexclusive, royalty-free right to publish or reproduce this article, or to allow others to do so, for Government purposes only. IMC'13, October 23-25, 2013, Barcelona, Spain.

Copyright 2013 ACM 978-1-4503-1953-9/13/10 ...\$15.00.

http://dx.doi.org/10.1145/2504730.2504745.

\section{Keywords}

DNS; Nameservers; Resolvers; IPv6

\section{INTRODUCTION}

After languishing for decades, IPv6 [13] is experiencing renewed interest [17], in large part due to economics and politics $[10,23]$. However, IPv4 and IPv6 are expected to coexist for the foreseeable future. Transition strategies, practical constraints, and inertia imply that portions of the IPv6 infrastructure will depend on IPv4, where hosts and infrastructure are "dual-stacked."

Previous measurement research has explored IPv6 penetration of the broader client population, for instance via web instrumentation $[11,32,27]$. Several initiatives [18, 19] and anecdotal evidence [28] suggest that IPv6 infrastructure deployment is proceeding in advance of client adoption. For instance, many large residential networks [12] and content providers [1] fully support IPv6. An important component of any provider's transition plans is enabling IPv6 on their Domain Name System (DNS) servers, including local recursive client resolvers.

Our motivation is interest in associating Internet DNS resolver IPv6 address(es) with IPv4 address(es). Operationally, such association is especially important to Content Distribution Networks (CDNs), where information of a client's recursive resolver is used to perform intelligent client routing and load balancing. We are particularly interested the simple case of one-to-one associations as such pairs are candidates for being on the same machine, or even interface, and thus collocated, which can be used as an input to IPv6 geolocation.

Our work is concerned with developing techniques for associating IPv4 and IPv6 DNS resolver addresses, and performing large-scale measurements to collect such associations. While out of scope for the present paper, obtaining DNS resolver associations between the two protocols has several potential future applications:

1. Internet Evolution: As IPv6 deployment continues, the infrastructure supporting each protocol will evolve. The techniques herein can be used in the future for a longitudinal study of tracking the inter-relationship of IPv4 and IPv6 nameservers.

2. IPv6 Geolocation: There is potential to leverage prior knowledge of geolocation of $\operatorname{IPv} 4$ addresses [5] to inform IPv6 geolocation when the addresses are correlated. One-to-one DNS resolver associations are con- 
sistent with, but not proof of, the corresponding IPv4 and IPv6 addresses being assigned to the same machine, and thus being at the same physical location. Further screening of such pairs, discussed in $\S 5.1$, can pick out those that are more likely to be collocated. However, as with other inputs to geolocation, our associations are likely to be noisy and thus must be appropriately weighted. Akamai Technologies is currently using screened output from the opportunistic passive technique, $\S 2.1$, in conjunction with prior knowledge of $\mathrm{IPv} 4$, as one of the inputs for IPv6 geolocation within its CDN.

3. Security: $\operatorname{IPv} 4, \operatorname{IPv} 6$ nameserver pairs that are likely on the same machine are of interest from a security viewpoint. Not only are many old IPv4 attacks feasible in IPv6 [7], IPv6 introduces new attack vectors [16]. An attack directed against the IPv6 address of a DNS server may also impact an organization's corresponding IPv4 service.

Our hope is that our techniques and data from this research can be used as a first step toward future work on these important applications.

To this end, we develop two novel techniques for discovering associated $\mathrm{IPv}_{\mathrm{v}} 4$ and IPv6 resolver addresses: i) a passive, opportunistic technique that uses a two-level DNS hierarchy that encodes IPv4 addresses within IPv6 nameserver records; and ii) an active technique whereby we probe resolvers to induce various lookup behaviors. Our techniques provide new methods to characterize a small, but critical, portion of the Internet infrastructure.

The passive technique has been implemented in the Akamai CDN, and for 674k (IPv4, IPv6) address pairs observed over a six month period, we find that $34 \%$ of the collected resolver addresses have a one-to-one association, i.e. appear in no other address pair. This percentage increases to almost $50 \%$ when the IPv6's are aggregated to /64 prefixes, (creating pairs of an IPv4 address with an IPv6 /64 prefix). We also discover complex, connected sets of address pairs that, at the extreme, span continents and hundreds of Autonomous Systems, where large cluster resolvers and public DNS providers play a major role. Additionally, we find evidence of abnormal use of 6to4 tunnels.

To help substantiate our results, we analyze the PTR records and software versions of the association inferences. For more precise validation of a subset of the associations, we employ our active measurement technique to perform a data collection over 13 hours to minimize temporal address assignment changes and corroborate the extant complexity discovered passively. Last, for samples sent to personal contacts at six major networks, three responded and manually confirmed that all of our inferred one-to-one associations that they checked were correct and indeed assigned to the same machine.

In total, this paper makes four primary contributions:

1. A new passive technique for opportunistically pairing IPv4 and IPv6 addresses of DNS resolvers.

2. A novel approach for active, targeted, DNS resolver probing to discover associated address pairs.

3. Real-world deployment of the passive technique on a commercial CDN to gather a large cross section of Internet resolvers.

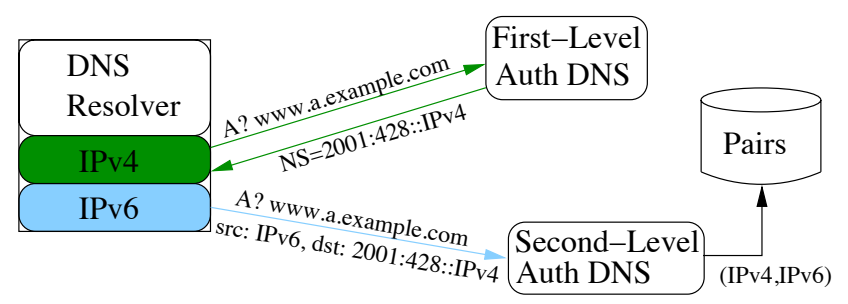

Figure 1: DNS Resolver IPv4/IPv6 Address Inference: A multi-level authority returns second-level nameserver AAAA records encoding a query's IPv4 source in the lower-order bits. The second-level nameserver associates IPv6 sources with the IPv4 encoded in the destination IPv6.

4. Analysis of observed DNS resolver address pairs that finds a significant proportion, $34 \%$, have a one-to-one association (almost 50\% when the IPv6's are aggregated to /64 prefixes) and also finds complex, connected sets of address pairs.

The remainder of this paper is organized as follows. Section 2 details the two measurement techniques comprising our system as well as our deployment. We summarize the collected data in $\S 3$, present its analysis in $\S 4$, and discuss findings in $\S 5$. Finally, we conclude in $\S 6$ with a summary of implications and suggestions for future work.

\section{METHODOLOGY}

Our system includes two novel measurement techniques. First, we develop a passive, opportunistic method to discover candidate IPv4 and IPv6 addresses of DNS resolvers. Second, we create a custom DNS server that permits active measurement of DNS resolvers. In this section, we detail these techniques.

\subsection{Opportunistic DNS Technique}

Clients rely on local recursive resolvers to perform DNS resolution. We seek to ascertain the IPv4, IPv6 addresses of dual-stacked DNS resolvers by inducing them to reveal their addresses as part of their natural lookup process.

Our technique exploits: 1) a two-level authoritative DNS resolution, and 2) the ability to encode an IPv4 address in the lower order bits of an IPv6 address. For the purpose of exposition, assume we control, and deploy our technique on, the DNS authority of example.com.

Consider a recursive DNS resolver servicing a local client requesting resolution of www.a.example.com. As depicted in Figure 1, the resolver requests resolution (typically an A record) for this host from the first-level authoritative nameserver via an IPv4 DNS query. The first-level nameserver responds with the second-level NS, and corresponding "additional" A and AAAA, records [22]. Crucially, the AAAA records of the second-level NS, as returned by our first-level nameserver, are formed dynamically. The first-level DNS encodes a query's IPv4 source address in the lower-order bits of the response's additional AAAA record.

For example, Figure 1 shows the first-level DNS responding to a DNS resolver's query with the authoritative NS record of the second-level DNS, including an additional AAAA record for the second-level nameserver that includes the querying resolver's IPv4 source address in the low-order bits. The 
recursive DNS resolver may use either the A or AAA address for the second-level resolution. When the latter is used, the second-level DNS can pair the IPv6 source address of the query it receives with the $\mathrm{IPv} 4$ address encoded in the IPv6 destination of the query. Note that the dynamically generated AAAA nameserver record is valid as the second-level DNS accepts queries from an entire prefix; we use a /80 prefix.

Akamai Technologies has deployed this passive technique within its production infrastructure [26], making use of its pre-existing multi-level DNS hierarchy (whose primary purpose is to resolve names to CDN addresses that can best serve the client), and has activated it for a selection of domains. Akamai has used the resulting data collection during the revision of its request-routing software to support IPv6, and continues to use it as one of the inputs into Akamai's geolocation product [5] where the extensive prior knowledge of IPv4 can be used to aid in the location of IPv6 when the IPv4 and IPv6 addresses are inferred to be correlated.

As a practical issue, the first-level authoritative DNS in Figure 1 does not know whether the client's nameserver is IPv6 capable, as the incoming DNS query arrives over IPv4. Thus, in Akamai's implementation, of eight NS records returned, only one or two of the associated glue records are AAAA. Also, even when the client's nameserver is dual stacked, it may not select the AAAA; selection order typically depends on historical load and performance characteristics.

Note that this technique is opportunistic: a client resolver must first contact our authoritative DNS for resolution of an object within our portion of the DNS namespace. No additional packets are sent when one deploys the technique for domain names that are being used anyway. Because the technique is passive, where the collection of address pairs is simply a by-product of the client's resolver requesting a domain under our authority, there are no restrictions on the population of resolvers we can measure. In particular, we are not limited to open resolvers as the entity collecting the data is not initiating the DNS query.

Also note that the passive technique only checks whether the client's resolver can send IPv6, but does not check whether it can receive IPv6. If it can not receive responses over IPv6, the query will timeout, and the resolver can fallback to retry using an $\mathrm{IPv}_{\mathrm{v}}$ glue record. In such a scenario, we still record an (IPv4, IPv6) pair. This ambiguity does not occur with the active technique of $\S 2.2$.

The passive technique can be implemented on any authoritative nameservers under common control along a DNS namespace hierarchy. It can be implemented even on a single machine, with multiple addresses, where the IPv4 glue address for the NS record for example.com is distinct from the IPv4 and IPv6 glue addresses for the NS record that causes the lookup to the second level. However, our deployment using separate first and second-level authorities is most compatible with the existing Akamai infrastructure, where deployment on Akamai affords us a rich and diverse dataset.

\subsubsection{Advantageous Complications}

Figure 1 illustrates the simple case where the DNS queries come from a single, dual-stacked machine. However, the reality of Internet DNS resolution can be much more complex. Recall that the (IPv4, IPv6) addresses that are logged are addresses seen by the authoritative nameservers. They need not be associated with the same machine. It is wellknown that DNS servers and resolvers experience significant load, and that many approaches exist for balancing DNS query load $[25,3,15,4]$. A relatively common case, especially among large providers, is that a provider maintains a set of servers at a location that share the load, and also may share a cache or may distribute obtained resolutions. Further, DNS resolver implementations may perform record pre-fetching [4] in an effort to improve performance.

In Akamai's implementation, the NS records returned by the first-level authoritative DNS have a TTL of 12 hours. Thus, if there is shared cache or if the results are distributed, then another nameserver may subsequently use cached results and send a query over IPv6, in which case the secondlevel nameserver will discover multiple IPv6 addresses associated with a single IPv4 address. A short TTL on the final A or AAAA record (20 seconds in the Akamai implementation) increases reuse of the cached NS record. Furthermore, after the NS TTL expires, if some other nameserver in the cluster does the lookup at the first-level, then another IPv4 address could be seen by the authoritative nameserver. In which case the data set will contain multiple pairs which have IPv4 or IPv6 addresses in common, all of which are associated with a given cluster of DNS servers at a given location. This complexity is an advantageous complication from a number viewpoints. For geolocation, one has discovered additional addresses that are likely collocated. For CDN's that wish to associate clients to nameservers, one might generalize an observed set of (client address, nameserver address) pairs to any of these clients potentially being associated with any of these nameservers. Further, from the viewpoint of survivability, or robustness, one has gained some insight into how the ISP has chosen to architect its DNS.

However, clusters of DNS servers complicate the discovery of pairs of $(\operatorname{IPv} 4, \operatorname{IPv} 6)$ addresses that can be inferred to exist on the same interface or machine. However, in [8], we describe preliminary work on an active fingerprinting technique that seeks to determine whether or not candidate address pairs are indeed on the same machine.

Another complication is when sometimes there is an intermediary machine between the client's nameserver and the authoritative first and second-level nameservers, as for example when a Network Operator has deployed a DNS cache hierarchy. Another case is when a public DNS, e.g. [2], is used for the IPv6 but not the IPv4, or vice versa. The presence of intermediary machines is a negative complication from the viewpoint of wishing to associate addresses to a common machine or set of machines at a given location. Though, it is interesting from the viewpoint of discovery of DNS architecture and how the DNS is being used, as discussed in $§ 4.1 .3$.

Note that when using the discovered (IPv4, IPv6) pairs for geolocation one needs to take into account that the data set will likely contain misleading information, as some of the pairs will have addresses that are in distinct locations. However, noisy inferences are not a new problem, but rather a well-known attribute of many of the inputs used for geolocation; and one must sort, weigh, and filter conflicting information from multiple sources.

\subsubsection{Example Nameserver Pairs}

To illustrate the potential complexity of the associations we gather passively, we present several example resolver pairs in this subsection. Often (to be quantified in §4.1.1) a given IPv4 address and a given IPv6 appear in only one pair. 
For example, the two addresses in the pair:

$(119.63 .216 .69, \quad 2402: 7400: 0: c:: 5)$

are only observed in this pair. However, just as common are more complex relationships. For example the IPv6 address $2001: 380: 515: 1:: 201$ is observed in two pairs:

$(210.227 .79 .198, \quad 2001: 380: 515: 1:: 201)$

$(210.227 .79 .230, \quad 2001: 380: 515: 1:: 201)$

and these two IPv4 addresses are only seen for this IPv6. Similarly, the IPv4 address 120.119.28.2 is observed in four pairs:

(120.119.28.2, 2001:e10:c41:49:c5e5:281b:44f8:80bd)

(120.119.28.2, 2001:e10:c41:51:226:b0ff:fedc:f970)

(120.119.28.2, 2001:e10:c41:1::2)

(120.119.28.2, $2001:$ e10:c41:59:cabc:c8ff:fe92:e8d6)

and these four IPv6 addresses are only seen in these pairs. The associations can become more involved, for example the following six pairs:

$(193.137 .16 .65, \quad 2001: 690: 2280: 1:: 65)$

$(193.137 .16 .65, \quad 2001: 690: 2280: 801:: 135)$

$(193.137 .16 .75, \quad 2001: 690: 2280: 801:: 135)$

$(193.137 .16 .75, \quad 2001: 690: 2280: 1:: 75)$

$(193.137 .16 .145,2001: 690: 2280: 801:: 135)$

$(193.137 .16 .145,2001: 690: 2280: 801:: 145)$

form a connected set of associations, in that starting at any address and following the associations, one would eventually touch each of the addresses in this set.

Such sets of address pairs can be complex. In the 6-month data set, Section 3, there is one particularly large group consisting of about a third of the 674,000 address pairs, and spanning hundreds of autonomous systems (ASes) and multiple continents. This large group occurs, at least in part, due to open, public nameservers such as GoogleDNS, and is of interest from the viewpoint of how DNS is being used, and is discussed in Section 4.1.3.

\subsubsection{Bipartite Graph and Equivalence Classes}

We abstract the observed resolver pairs as a bipartite graph between a set of $\mathrm{IPv} 4$ addresses and a disjoint set of IPv6 addresses. Each of the observed address pairs is an edge of the graph connecting a vertex in the IPv4 set to a vertex in the IPv6 set. The graph is not fully connected, but rather consists of many subsets of connected edges.

We call a set of connected edges (and the address pairs they represent) an "equivalence class," abbreviated "eq. class." Figure 2 illustrates an example bipartite graph consisting of five $\operatorname{IPv} 4$ addresses, seven IPv6 addresses, and eight address pairs (edges). Let $m$ - $n$ denote an equivalence class containing $m \operatorname{IPv} 4$ and $n \operatorname{IPv} 6$ addresses. In Figure 2 the address pairs partition into four equivalence classes, two of which are $1-1$, one is $2-1$ and one is $1-4$.

\subsection{Active DNS Measurements}

In addition to the opportunistic, passive DNS data collection, we develop an active DNS measurement to both validate and better understand our passive results.

We use this active technique to probe the subset of passively gathered resolvers that respond to external requests, e.g. the subset containing open and forwarding resolvers [6].

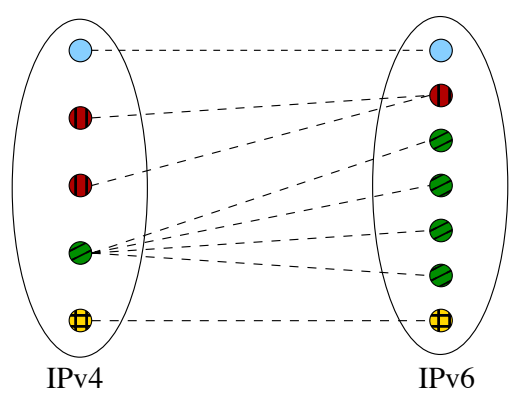

Figure 2: Equivalence Classes: The associations (edges) between IPv4 and IPv6 addresses form a bipartite graph. The connected components form equivalence classes of various sizes. In this hypothetical example, the nodes in each equivalence class are shaded uniquely. Two of the four equivalence classes are 1-1 (one IPv4, one IPv6 node), one is 2-1, and one is $1-4$.

More generally, this technique can be used on any resolver that will respond to probes from one's vantage point, such as those of one's own network, or the numerous open DNS forwarders on the public Internet.

One actively probes the resolvers issuing specially crafted queries for DNS records for which we are authoritative. In this way, we control both the DNS probes and the authority of the DNS records being probed, thereby permitting testing of open DNS recursive resolvers in our dataset. Figure 3 depicts the high-level active DNS probing methodology.

Our authoritative domains are served by a custom DNS server that is standards compliant [14]. Our authoritative server listens on both IPv4 and IPv6, but returns different results depending upon the incoming request. The server handles multiple domains that support either IPv4 or IPv6 requests, where the choice of domain impacts the IP protocol used by a recursive resolver.

We initiate queries to the open and forwarding resolvers. The results from our DNS server for the queried object induce the resolver under test to issue a series of queries that alternate between IPv4 and IPv6 for network transport. We maintain state between requests by specially encoding the returned results such that the final response to the recursive query is a "chain" of IPv4 and IPv6 addresses used by the resolver under test. Mao et al. [21] also encode an Internet address in a domain name, though in the different context of associating $\mathrm{IPv} 4$ clients with the client's recursive resolver address, and where a web bug is inserted in volunteer web pages. Figure 4 provides an example timing diagram of the interaction of our prober and an authoritative DNS server with an open resolver whose addresses we wish to infer. In this example, the resolver has IPv6 addresses $=A 1, A 3$ and IPv4 addresses $=A 2, A 4$.

The prober queries the open resolver or forwarder for a single TXT record. The resolver can only fetch this name using IPv6, but instead of returning the record's value, our server returns a canonical name (CNAME) alias. This CNAME encodes the IPv6 address which contacted our server; for example an IPv6 address $2001: f 8 b 0:: 91$ is encoded into the CNAME:

2001yf8b0yy91.v4.dnstest.icsi.berkeley.edu 


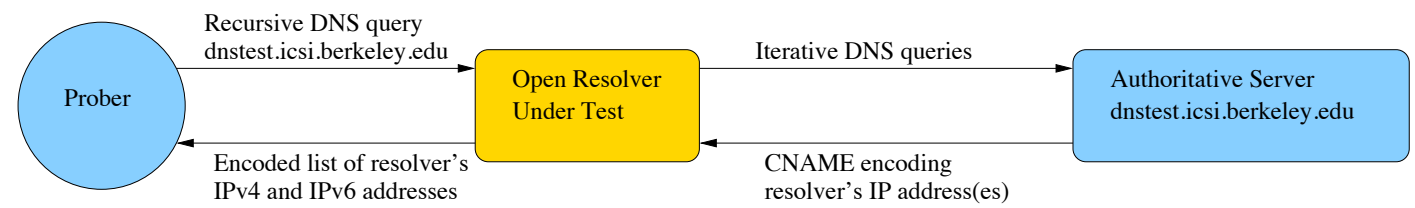

Figure 3: Active DNS Probing: We send queries for DNS records whose authority is under our control (shaded blue), thereby permitting testing of open resolvers in our dataset. Our authoritative server is specially designed to: 1) force the recursive resolver to alternate between IPv4 and IPv6 transport; and 2) encode the resolver's source IP addresses. The result of our DNS probes is an encoded list of the resolver's IPv4 and IPv6 addresses.

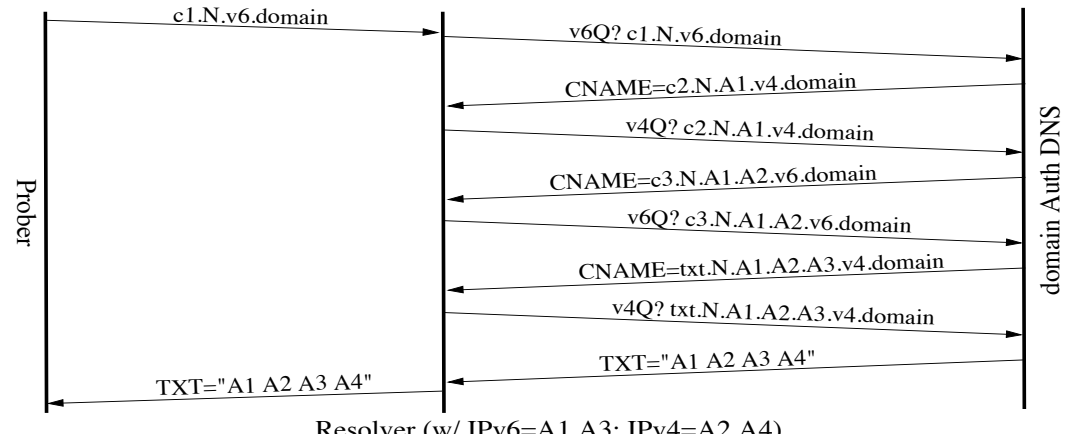

Resolver (w/ IPv6=A1,A3; IPv4=A2,A4)

Figure 4: Active DNS Probing: Our authoritative DNS server returns a series of CNAME results with alternating IPv6 and IPv4 glue. We probe a resolver under test for our special domain, including a nonce $N$. State is maintained on addresses the resolver uses by encoding the IPs along the chain. The final result is the sequence of IPv4, IPv6 addresses used by the resolver (here $A 1, A 2, A 3, A 4)$.

This returned CNAME exists within the IPv4-only domain. The next CNAME redirects back to IPv6, encoding both IPs. After following another CNAME back to the IPv4 domain, our server finally returns a TXT record reporting the sequence of four IP addresses which contacted our server. Note that while DNS authority servers may typically include multiple records in a single returned result, our server only returns one result at a time in order to force multiple lookups and infer the chain. Our CNAME encoding scheme, combined with DNS message compression [22], ensure that, even in the worst case ASCII IPv4 and IPv6 encoding expansion, our chains of length 4 are less than 512 bytes. As $512 \mathrm{~B}$ is the limit for DNS over UDP, we ensure that our chains rely on neither truncation nor EDNS0 [30].

Note that our methodology includes several techniques to ensure accuracy. First, each query includes a nonce that prevents effects due to DNS caching. Second, all state is maintained in the queries themselves, thereby removing the potential for miscorrelation of $\mathrm{IPv} 4$ and IPv6 addresses. For example, to infer a set of IPv4 and IPv6 addresses used by the Google public DNS resolvers, we query:

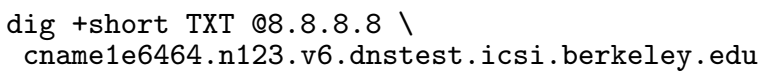

where the Google DNS resolver, after stepping through the CNAME's, finally sends a query for the TXT record:

txt.n123.2607yf8b0y4004yc00yy153.64x233x168x86. I 2607yf8b0y4004yc00yy156.v4.dnstest.icsi.berkeley.edu.

The authoritative DNS, notes the three addresses contained in the requested domain, and notes the source address of the incoming query. The authoritative DNS then returns the TXT record that includes the nonce and the sequence of addresses that contacted our server to resolve the request; in this example:

"n123" ।

"2607:f8b0:4004:c00: : 153" "64.233.168.86"।

"2607:f8b0:4004:c00::156" "64.233.168.85"

As we will show in $\S 4$, many large-scale resolvers are actually clusters, not individual systems. A cluster might be behind a single publicly facing IP address with load distributed among multiple backend machines, or might encompass multiple publicly visible IP addresses. Thus, one can repeat the active DNS probes multiple times in order to gain a more complete picture of cluster structure when present. Since the DNS specification [14] requires that the recursive resolver process the entire CNAME chain, these four IP addresses should represent the same "system" responsible for completing the DNS resolution. ${ }^{1}$ The replies themselves have a 0 second TTL and the request contains a counter, thus a resolver should never cache the result.

\footnotetext{
${ }^{1}$ We initially noticed a complication where a NAT forwarder could send an initial lookup to our server but, after receiving our CNAME reply, it queries instead a configured recursive resolver for the CNAME, as the initial IPv4 address was only used in the first of the two IPv4 queries and did not otherwise cluster well with the rest of the resolution change. We speculate that most such systems are not IPv6 capable, so we changed our query's order from V4/V6/V4/V6 to $\mathrm{V} 6 / \mathrm{V} 4 / \mathrm{V} 6 / \mathrm{V} 4$ to prevent a NAT from initiating the request directly, forcing it to contact the configured recursive resolver first to complete the resolution process.
} 


\begin{tabular}{|l|l|l|l|l|l|l|}
\hline Set & Method & Collection Period & $\begin{array}{l}\text { Num. } \\
\text { IPv4 }\end{array}$ & $\begin{array}{l}\text { Num. } \\
\text { IPv6 }\end{array}$ & $\begin{array}{l}\text { Num. } \\
\text { Pairs }\end{array}$ & Notes \\
\hline $\begin{array}{l}\text { 6-month } \\
\text { data set }\end{array}$ & Passive & $\begin{array}{l}\text { Mar. 17 to Sep. 13, } \\
2012\end{array}$ & 270,000 & 282,000 & 674,000 & $\begin{array}{l}\text { From Akamai's production deployment. Includes first } \\
\text { and last time the address pair was recorded. }\end{array}$ \\
\hline $\begin{array}{l}\text { 12-day } \\
\text { data set }\end{array}$ & Passive & Apr. 18 to 30, 2012 & 47,000 & 46,000 & 119,000 & $\begin{array}{l}\text { From Akamai's production deployment. Includes the } \\
\text { number of times an address pair was recorded. }\end{array}$ \\
\hline $\begin{array}{l}\text { 1-day } \\
\text { data set }\end{array}$ & Active & Apr. 19 to 20, 2013 & 5,000 & 8,000 & 41,000 & $\begin{array}{l}200 \text { repeated tests to 7,000 open resolvers of the 6- } \\
\text { month data set. }\end{array}$ \\
\hline
\end{tabular}

Although limited to probing IPv6-capable open resolvers and forwarders (thus excluding most corporate networks), the active measurement has several advantages over the passive measurements. This technique forces the resolver to use IPv6 (instead of relying on a resolver's preference for IPv6 over IPv4). Since the measurements all occur within a short time window, this measurement is not affected by network changes. It also produces a set of up to four associations, allowing it to more effectively and precisely map the structure of a cluster resolver.

\section{DATA SETS}

We examine three data sets, summarized in Table 1 . The 6-month data set is from Akamai's production deployment. The address pairs are obtained by the passive, opportunistic DNS technique, described in $\S 2.1$. Akamai observes a significant cross-section of global DNS traffic in its role as a large CDN; the dataset includes resolvers from over 213 countries [5] and contains: 674,000 unique (IPv4, IPv6) pairs with 271,000 unique IPv4 and 282,000 unique IPv6 addresses.

The 12-day data set is also from Akamai's deployment and the passive DNS technique, though the logs were collected and aggregated via a script that records the number of observations of each address pair. A measure of the popularity of an address pair is useful as an additional criteria when screening for collocated addresses, and it is being incorporated into a revised collection and aggregation of the Akamai logs.

The 1-day data set is obtained by the active technique of $\S 2.2$. We test the addresses in the 6 -month data set to determine whether they return a response from our prober, i.e. whether they are open ${ }^{2}$, and we receive responses from 5,308 IPv4 and 1,677 IPv6 resolver addresses. While this is only a subset of the full data, it permits validation against a meaningful pool of systems. These addresses are then repeatedly probed, at least 200 times over the next hours. ${ }^{3}$ We use this active data set to investigate the additional complexity in the DNS infrastructure that is revealed by repeated probes to a given set of resolvers.

\footnotetext{
${ }^{2}$ We again emphasize that while our active technique requires an open resolver, the passive technique does not.

${ }^{3}$ The returned v6/v4/v6/v4 4-tuple often contains addresses distinct from the addresses probed, thus, from $\approx 7,000$ open resolvers probed, $\approx 13,000$ addresses occur in the 4 -tuples, Table 1. The number of systems probed is significantly lower than the number of open "recursive resolvers" because this only included systems supporting IPv6 and only included true open resolvers, rather than the bulk of misconfigured home NATs and other DNS forwarders.
}

\section{RESULTS}

This section analyzes results from deploying the aforementioned techniques on the $\mathrm{IPv} 4$ and $\mathrm{IPv} 6$ Internet. We first examine the six-month passive data set, focusing on the 1-1 eq. classes, and the ability to limit the prevalence of complex associations via various forms of aggregation. To better understand the remaining complexity, which is a small fraction of the eq. classes, but a large number of addresses, we examine one of the largest eq. classes. Using our active technique, we exploit the subset of open resolvers in the six-month passive data set to show similarly complex eq. classes. We then consider the additional attribute of the frequency that an address pair is observed. Last, we perform three forms of validation to increase the confidence in our results: probing for consistent software version and PTR records, and manual verification by network operators.

\subsection{Passive Technique Address Discovery (Six- Month Data Set)}

We examine the address pairs of the 6-month data set. Recall that we define an "Equivalence Class" to be a set of connected edges (address pairs) in a bipartite graph (§2.1.3). A $m-n$ eq. class consists of $m \operatorname{IPv} 4$ and $n \operatorname{IPv} 6$ addresses, and a 1-1 eq. class is the simple case of an address pair where neither address has been observed in any other pair, and is consistent with (but not a guarantee of) the two addresses being assigned to a common resolver.

Figure 5 is a scatter plot of all eq. classes, using their relative frequency as the color key. We observe that the small eq. classes, 1-1 and 1-2, are the most common, and that there exists a broad range of sizes, with larger eq. classesoften representing a singular occurrence. We focus first on the 1-1 eq. classes, and then examine the larger ones.

\subsubsection{Focus on 1-1 Equivalence Classes}

The canonical case of a simple dual-stack server with a single routable IPv4 and IPv6 address would be observed as a 1-1 eq. class, though the converse need not hold as a 1-1 relationship may also be the public facing portion of a more complicated architecture. From the perspective of finding candidate dual-stack servers more 1-1 eq. classes is better.

As shown in the first row of Table 2, 34\% of the addresses (IPv4 plus IPv6) of the 6-month data set are in 1-1 eq. classes. Although, not a majority, it is still a substantial portion, and increases to almost $50 \%$ when we consider aggregation to prefixes.

\section{Aggregation to Prefixes.}

Frequently, multiple addresses of a non-1-1 equivalence class reside in a given network prefix. Therefore, for each equivalence class, we examine aggregating addresses by pre- 


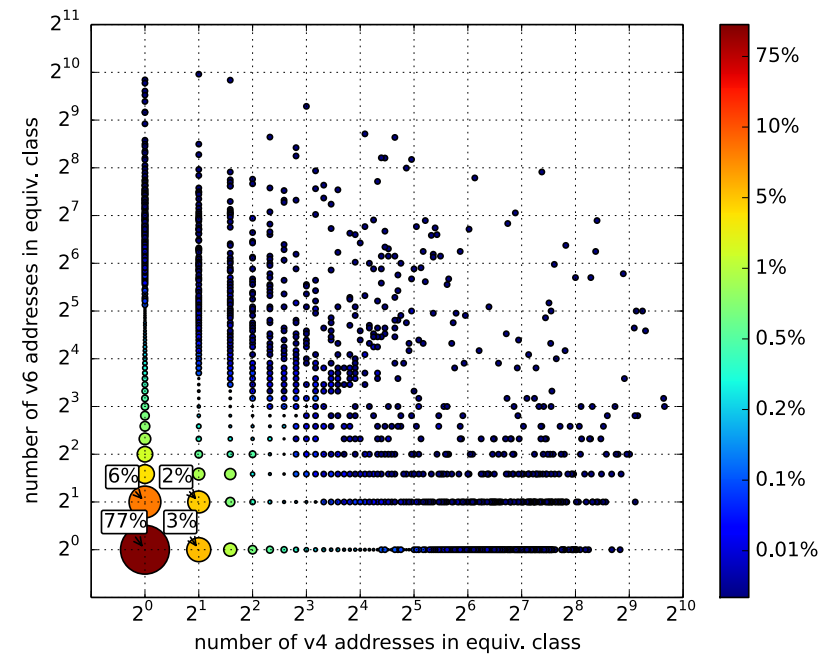

Figure 5: Scatter plot of number of IPv4 and IPv6 addresses in the equivalence classes. Only the 1-1, 1-2, 2-1, and 2-2 eq. classescomprise more than $1 \%$ of the total population, however there are a small number of large eq. classesof varied sizes.

fix, thereby forming network-specific eq. classes. There is a trade-off regarding the chosen size of the prefix, where larger prefixes lead to more 1-1 eq. classes, but also increase the chance of pooling together unrelated equipment. For example, aggregating to prefixes as advertised by BGP would produce eq. classes with a natural association to the networks. However, the Network Operator that advertises a given prefix is not claiming that the addresses therein are located in a given Point of Presence, and are unlikely to be so for larger prefixes.

Here, we concern ourselves with 1-1 eq. classes whose addresses are likely candidates to be collocated. Therefore, we consider smaller prefixes to increase the chance of collocation. For IPv6, a natural choice is to aggregate across the interface ID in the lower 64 bits. Established practice is for IPv6 addresses in a given /64 to be on a common subnet, or may even be associated with a single interface where the interface ID is varied for anonymity, [24]. For IPv4 there is no natural choice for aggregation. Commonly, not always, operators assign to servers in a given rack (or subnet, or building) addresses that are close to one another in address space. A reasonable assumption (but for which there would be exceptions) is that addresses of servers in the same /30 would be in the same building. Assuming a /29 is a somewhat more aggressive, and so on. Fortuitously, given the arbitrariness in choice of prefix, we find that for the 1-1 eq. classes there is an insensitivity, at least up to /24's and /48's.

As an example of aggregation, the following five pairs:

$(122.1 .94 .240,2001: 380: 150:: 1053)$

$(122.1 .94 .240,2001: 380: 11 \mathrm{c}:: 1053)$

$(122.1 .94 .240,2001: 380: 11 \mathrm{c}:: 2053)$

$(122.1 .94 .242,2001: 380: 11 \mathrm{c}:: 1053)$

$(122.1 .94 .243,2001: 380: 11 \mathrm{c}:: 2053)$
Table 2: Prevalence of 1-1 equivalence classes in the 6-month data set

\begin{tabular}{|l|l|l|}
\hline Data Set & Entity & $\begin{array}{l}\text { \% } \\
\text { IPv4+IPv6 } \\
\text { addresses in } \\
\mathbf{1 - 1} \text { eq class }\end{array}$ \\
\hline 6-month data set & addresses & $34 \%$ \\
\hline 6-month data set & prefixes - , /64 & $47.6 \%$ \\
\hline 6-month data set & prefixes $/ 30, / 64$ & $47.9 \%$ \\
\hline 6-month data set & prefixes $/ 27, / 64$ & $48.2 \%$ \\
\hline 6-month data set & prefixes $/ 24, / 64$ & $48.6 \%$ \\
\hline 6-month data set & prefixes $/ 24, / 48$ & $49.7 \%$ \\
\hline 6 -month data set & prefixes $/ 16, / 32$ & $62.4 \%$ \\
\hline 6 -month data set & AS's & $80 \%$ \\
\hline \hline $\begin{array}{l}\text { Restrict to final } \\
\text { week }\end{array}$ & addresses & $71 \%$ \\
\hline $\begin{array}{l}\text { Random subset; } \\
\text { same num of pairs } \\
\text { as in last week }\end{array}$ & addresses & $51 \% \pm 0.5 \%$ \\
\hline \hline Example in Fig 2 & addresses & $33 \%$ \\
\hline
\end{tabular}

form a 3-3 eq. class of addresses. When aggregated to prefixes /30, and /64 they become:

(122.1.94.240/30, 2001:380:150::/64)

(122.1.94.240/30, 2001:380:11c: :/64)

a 1-2 eq. class of prefixes /30, /64.

For a more dramatic example, paired with 88.191 .68 .83 are $101 \operatorname{IPv} 6$ addresses. All of the IPv6 addresses are in the single prefix $2 \mathrm{a} 01: \mathrm{e} 0 \mathrm{~b}: 1: 68 / 64$. The 6 -month data set includes timestamps of when a given pair is first and last seen, and these IPv6's were almost always observed in disjoint 24-hour periods. This is likely an example where there is a single interface and the interface identification (the 64 lowest order bits) varies. Thus, whereas the addresses form a 1-101 equivalence class, after aggregating the IPv6's to /64 prefixes, we obtain 1-1 eq. class of prefixes.

The second through seventh rows of Table 2 report the aggregation to prefixes for different masks, where the equivalence classes are now of prefixes. When aggregating just the IPv6's and to /64's, the second row, the percent of addresses that are in the 1-1 eq. class jumps from $34 \%$ to $47.6 \%$. When we also aggregate the IPv4's to prefixes, there is only a minor increase in the percentage even for aggregation to /24 and /48 respectively, which is rather aggressive from the viewpoint of addresses that are likely to be on the same subnet or location. (Aside: the choice of prefix has a more noticeable impact for eq. classes greater than 1-1; see $\S 4.1 .2$.) Out of curiosity, when we do an extreme aggregation to $/ 16$ and $/ 32$ prefixes, we get that $62 \%$.

In summary, from the viewpoint of finding (IPv4, IPv6) address pairs that are candidates for being collocated, we observe that aggregating the IPv6's to /64's yields almost $50 \%$ of the addresses to be in the $1-1$ equiv. class, and that there is little need for the somewhat more dubious step of also aggregating the IPv4's. See $\S 5.1$ for further comments.

\section{Aggregation to AS's.}

Shifting the focus from addresses that are candidates for being collocated, and to consider their distribution across networks, we consider aggregation to AS's. We exclude pairs where the IPv6 address is 6to4, as such addresses have ambiguous AS assignments. With 6to 4 excluded, $80 \%$ of the 
addresses are in the 1-1 eq. class of AS's, Table 2. However, this includes the circumstance when the two AS's are not equal to each other. (This issue does not arise for prefixes, as the $\mathrm{IPv} 4$ is always distinct from the IPv6.) When the two AS's are different, by far the most common case is where the IPv6 AS is 6939, the tunnel broker Hurricane Electric. If we remove the address pairs of Hurricane Electric, then $51 \%$ of the remaining addresses are in the 1-1 of AS's where we impose the additional condition that the two AS's are equal to each other. Of the remaining 1-1 eq. classes of AS's where the two AS's are different, often the two AS's belong to the same organization. For example, of these AS pairs with distinct elements, the most popular AS is 7018, ATT. And of the AS's that are paired with $7018,82 \%$ are AS 7132, SBISAS ATT Internet Services, and another 9\% are AS 6389, BellSouth.net Inc. As an example of different organizations, when AS 3320, Deutsche Telekom, is in a 1-1 eq. class with a different AS, the most popular other AS is 8422 NetColonge, which suggest business relations besides what can be inferred from interconnection.

\section{Restriction to a Shorter Observation Interval.}

As the data set is a collection over a six month period, some address assignments and configurations likely changed during this period. These changes are reflected as larger eq. classes, as additional complexity, when in actuality, these large eq. classes may not have ever existed at a given moment in time. In this section, we examine this effect by considering a shorter observation interval.

However, when we restrict to a shorter time interval, we actually introduce two effects that reduce the larger eq. classes, and increase the proportion of addresses in the 11 eq. class: 1) less opportunity for changes in address assignments; and 2) a smaller data set. With a smaller data set, there is less opportunity for extant complexity to be revealed. (Imagine the extreme case of just a few observations, in which case large eq. classes are not possible.)

In order to separate these two effects, we also take random subsets of the full data set, where the subsets have the same number of pairs as present in the shorter observation interval. As the random subsets have address pairs from the full, 6-month observation interval, they exhibit just the first effect of a smaller data set.

The 6-month data set includes the time epoch the given pair was last observed. Thus, we can pick a time period, and then examine the subset of the pairs that are last observed during that period. We pick the length to be a week, which is short enough that the opportunity for address reassignments is much less as compared with 6 months, and long enough that we get an appreciable number of pairs, to somewhat mitigate the impact of the smaller data set. Also, we pick the week to be final week of the 6 -month period, as these are the pairs that are most active, and there are two to eight times as many pairs, 83,000, as compared with any other week on the six months.

Table 2 shows the results for the restriction to the final week, as well as for the random subsets, where for the latter, 10 subsets were taken and the table reports the mean values and the range. Table 2 shows that the fraction of addresses in the 1-1 eq. classes increases from $34 \%$ to $51 \%$ when we take the random subsets, which is the effect of a smaller data set. And the percentage increases further to

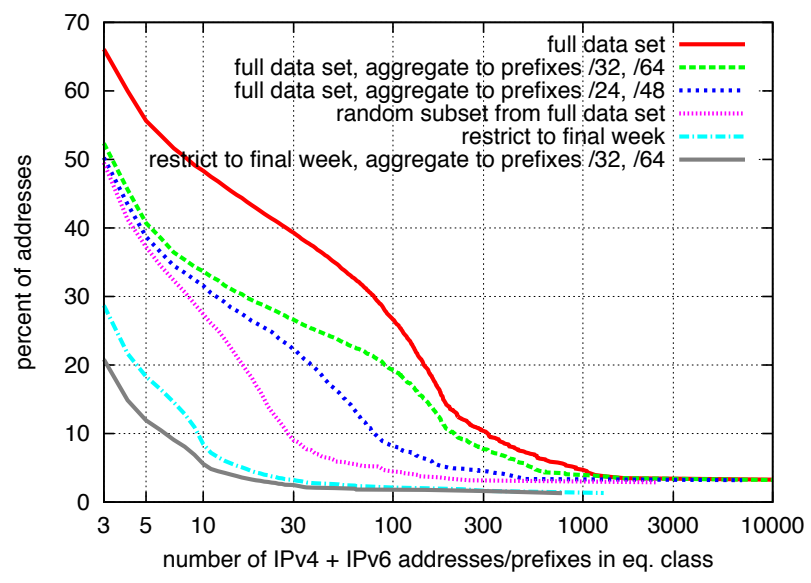

Figure 6: Percent of IPv4 + IPv6 addresses in equivalence classes of at least a given size

$71 \%$ when we restrict to the final week, where the increment from $51 \%$ is the effect of reduced opportunity for changes in configuration.

\subsubsection{All Equivalence Classes}

In this and the next three sub-sections, we shift the focus from the 1-1 eq. classes, and candidate address pairs that likely are on the same machine or subnet, to the non-1-1 eq. classes.

Figure 6 shows the percent of addresses that are in eq. classes of at least a given size, where "size" is the sum of IPv4 plus IPv6 addresses (prefixes) in the eq. class. One can view Figure 6 as the complementary distribution of an address being in an equivalence class of a given size. For example, looking at the full data set, the red line, $40 \%$ of the addresses are in equivalence classes of size at least 27. While, when aggregating IPv6 to /64 prefixes, the green line, $40 \%$ of the addresses are in equivalence classes (of prefixes) of size at least 5 . Note that with the $\mathrm{x}$-axis beginning at 3 , one can infer the percent of addresses in 1-1 eq. classes, e.g. the $y$-intercept of $66 \%$ means that $34 \%$ of the addresses are in an eq. class of size 2, as reported in Table 2. The curves that are higher up in the Figure indicate that, overall, the equivalence classes are larger and more complex - the distribution has a heavier tail.

Figure 6 shows that although the aggregation of IPv6 to /64 noticeably reduces the tail of the distribution, still $20 \%$ of the addresses are in eq. classes of size 90 or more. As expected, with greater aggregation, to /24 and /48 prefixes, the tail of the distribution is further reduced. A more substantial reduction occurs when we take a random subset from the full data set, where the number of pairs in the subset equals the number of pairs observed in the final week, $\S 4.1 .1{ }^{4}$ This reduction is due to the smaller sample size as compared to the full data set. That is, the additional observations in the full data set reveals substantial more complexity. We use the active technique of $\$ 2.2$, to perform a more controlled data collection, over a short time period, and examine the additional complexity discovered with additional probing in $\S 4.2$. When we restrict the data to the final

\footnotetext{
${ }^{4}$ The plots for the different random subsets are very similar to one another.
} 
week, there is a significant reduction as compared with the random subset. Although the random subset has the same number of address pairs, it covers a period of six months, and thus there is a greater opportunity for changes in address assignments and configurations. Lastly, as expected, there is somewhat further reduction when the IPv6's are aggregated to $/ 64$ 's for the eq. classes of the final-week data set.

In all of the cases of Figure 6, there is a long tail. This is due to the tendency for there to be one eq. class that is much larger than the others, as discussed next.

\subsection{3 "Mammoth" equivalence class}

Here, we examine the largest equivalence class. As mentioned in $\S 2.1 .2$, one of the equivalence classes is huge, 8037 IPv4 addresses and 9582 IPv6's, 8037-9582, which we call the "mammoth" eq. class - it contains $3 \%$ of the addresses and $37 \%$ of the address pairs. (The next largest eq. class contains $0.3 \%$ of the addresses and $0.2 \%$ of the pairs.) This huge eq. class, whose addresses span multiple continents and are in over $750 \mathrm{AS}$ 's, is of interest from the viewpoint of how DNS is being used and architected, and how such a diverse set addresses could be associated with one another (and clearly does not represent a set of load balanced nameservers at a single location).

The much larger percent of pairs, $37 \%$, as compared with percent of addresses, $3 \%$, occurs because a minority of the addresses are in many pairs. In terms of the bipartite graph, some of the vertices have many edges.

Figure 7 shows the complementary degree distribution of the vertices in the mammoth eq. class. That is, the percent of addresses (vertices) in the mammoth eq. class that are in at least a given number of pairs (edges). Figure 7 shows that the degree distribution for the IPv4 vertices is roughly the same as for the IPv6, though with a somewhat heavier tail. Although $54 \%$ of the addresses are in just one address pair, i.e. have degree one,$^{5}$ as seen from the y-intercept, $8 \%$ of the addresses are in at least 100 pairs, $4 \%$ in at least 300 pairs, and $1 \%$ in at least 500 pairs. As a comparison, for the full 6-month data set $89 \%$ of the addresses have degree one, and only $1 \%$ have degree at least 15 .

AS 15169, Google, plays a major role in this mammoth eq. class. ${ }^{6}$ Although just $9 \%$ of the addresses in the mammoth eq. class are in AS $15169,90 \%$ of the address pairs have one or both address(es) in that AS. $97 \%$ of the addresses that appear in at least 100 pairs are in AS 15169.

Whenever the IPv6 address is in AS 15169, then so is the paired IPv4. However, whenever the IPv4 address is in 15169 , the paired IPv6 is not quite always in $15169-1 \%$ of the IPv6's are 6to4 and another $1 \%$ are scattered across a hundred different AS's. This is how addresses outside AS 15169 become a part of this eq. class. Some of these non15169 IPv6 addresses may still be a part of Google's DNS architecture, assuming that Google has deployed servers in local ISP's with that ISP's addresses. And also, some may be separate from GoogleDNS - if the client's nameserver, using the public GoogleDNS for IPv4, obtained and cached the NS

\footnotetext{
${ }^{5} \mathrm{~A}$ degree one vertex in the bipartite graph does not imply that the vertex is in a a 1-1 eq. class. as the paired vertex can have degree greater than one.

${ }^{6}$ Other third-party DNS providers, such as OpenDNS and DynDNS, are present in the data set, but to a much lesser extent compared with GoogleDNS.
}

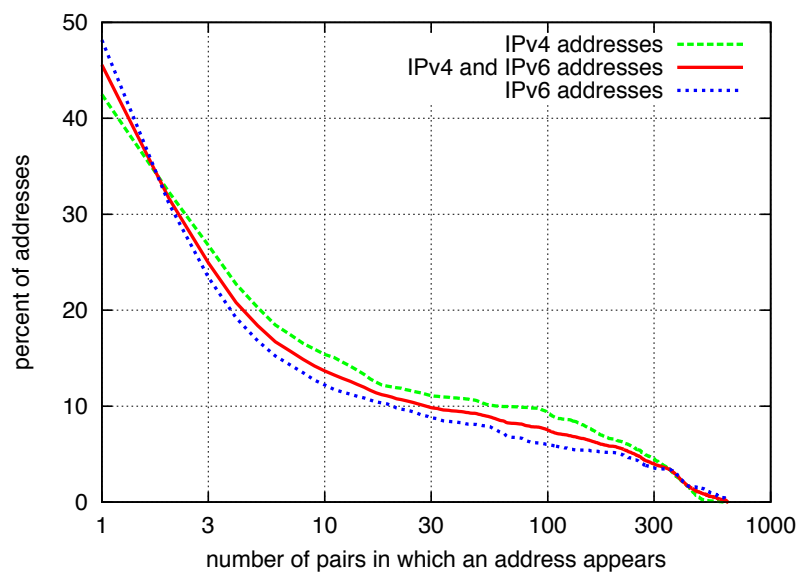

Figure 7: Percent of addresses in the "mammoth" eq. class that are in at least a given number of pairs. (Complementary distribution of node degree in bipartite graph of "mammoth" eq. class.)

Table 3: Impact on the largest equivalence class of the 6-month data set when selected address pairs are removed

\begin{tabular}{|c|c|c|c|}
\hline \multirow[b]{2}{*}{ Action } & \multicolumn{3}{|c|}{$\begin{array}{l}\text { After action, recompute eq. classes. } \\
\text { Resulting largest eq. class contains: }\end{array}$} \\
\hline & $\begin{array}{l}\text { Num of } \\
\text { IPv4 } \\
\text { addr. }\end{array}$ & $\begin{array}{l}\text { Num of } \\
\text { IPv6 } \\
\text { addr. }\end{array}$ & $\begin{array}{l}\text { Num of } \\
\text { addr. } \\
\text { pairs }\end{array}$ \\
\hline $\begin{array}{ll}\text { no change to } \\
\text { data set }\end{array}$ & 8,000 & 9,600 & 247,000 \\
\hline omit 6to4 & 4,000 & 5,100 & 230,000 \\
\hline omit AS 15169 & 5,600 & 4,900 & 18,000 \\
\hline $\begin{array}{l}\text { omit } 6 \text { to4 and } \\
\text { AS } 15169\end{array}$ & 900 & 2,000 & 3,400 \\
\hline $\begin{array}{l}\text { random subset } \\
\text { from full data } \\
\text { set; same num- } \\
\text { ber of pairs as } \\
\text { prior line }\end{array}$ & $2,623 \pm 214$ & $2,626 \pm 335$ & $78,778 \pm 603$ \\
\hline
\end{tabular}

records returned by the first-level authoritative nameserver; and then for subsequent resolutions, sent a DNS query over IPv6 to the low-level authoritative nameserver.

6 to 4 addresses also play a significant role in the mammoth eq. class. Although 6 to 4 addresses are in only $6 \%$ of the pairs, they are $42 \%$ of the IPv6 addresses in this eq. class (an imbalance that is the opposite of AS 15169). Interestingly, for many of the 6 to 4 addresses, the embedded IPv4 address does not equal the observed paired IPv4. See $\S 4.1 .4$ which discusses 6 to 4 in the full data set, not just the mammoth eq. class.

It is of interest to see what becomes the largest eq. class when, say, AS 15169 addresses are removed. Note that if one removes a set of addresses from the mammoth eq. class, the mammoth eq. class can partition into multiple smaller eq. classes - the remainder need not be a connected set of edges in the bipartite graph. Thus, we start with the full data set, and remove pairs that contain the addresses in question, and then with the reduced set of pairs, we recompute the equiv. classes and then note which is the largest one. Table 3 shows the results for selected removals. 
As discussed in Section 4.1.1, some of the reduction is simply due to a smaller number of address pairs. Thus, ten random subsets from the full 6-month data set are taken, where the number of pairs in the subset is the same as in the fourth line of the Table, 225,000 pairs, down from 674,000. Given that the cases of the fourth and fifth line have the same number of pairs, their differences are due the selective removal of particular pairs, the fourth line, versus random removal. The mean value and range is reported in fifth line, and shows that the major cause for the lower complexity reported in line four is not due the smaller sample, but rather the selected pairs that are omitted.

Even with the omission of 6 to 4 and AS 15169, the resulting largest eq. class still has thousands of address pairs. In $\S 4.3$ we continue the examination of largest eq. classes where we consider the 12-day data set, which has the additional information of the popularity of an address pair, and we show that significant further simplification is obtained when we omit infrequently observed pairs.

\subsubsection{Abnormal behavior with 6 to 4}

Auto-tunneled [9] addresses raise a natural question whether the embedded IPv4 address (i.e. the 32 bits that come after the "2002:") matches the paired IPv4 address from the discovery technique. Somewhat surprisingly, in $63 \%$ of the pairs where the IPv6 address is 6to4, the embedded IPv4 address is different from the paired address. Overall, any 6 to 4 traffic is probably a bug: Given a choice between an IPv4 address and a 6 to 4 address, a host should always prefer IPv4 unless executing a "happy eyeballs" strategy. Since the resolver also receives an $\mathrm{IPv} 4$ glue record, the resolver should prefer the IPv4 glue record, never attempting to connect to the DNS authority using 6to4.

Sometimes the 6to4 address is paired with hundreds of IPv4's. For example, 2002:aca8:101: :aca8:101 appears with 270 IPv4's. The embedded address is 172.168.1.1, on AOL, and located in the USA. The paired IPv4's are mostly in Brazil, but also Germany, Saudi Arabia, and Turkey.

A likely explanation is some sort of buggy resolver software with a hardcoded 6 to 4 address. That we receive such 6 to 4 traffic also suggests that the 6 to 4 gateway processing this traffic isn't validating that the embedded IPv6 address corresponds to the $\mathrm{IPv} 4$ address. It is not likely to be an attack as, if an attacker took advantage of this, the query the attacker would employ would cause significantly more amplification than the name lookups present in an Akamai-ed domain.

Also, there are stranger cases where the 6to4 is again paired with many IPv4's but the embedded IPv4 is not routable. For example 2002:6464:64cd::6464:64cd is paired with over 250 IPv4's and the embedded address is 100.100.100.205 or 2002:10::1:219:dbff:fef9:aa4f with embedded address 0.16.0.0. Again, this suggests buggy software misusing 6 to 4 .

Although further investigation would be needed for a definitive explanation, it is clear that the collection of nameserver associations has turned out to have the additional feature of revealing abnormal activity.

Note: in calculating the percent of addresses that are in one-to-one associations, $\S 4.1 .1$, we kept all of the complexities in the data set, including the abnormal associations. To the extent we remove any, the percentages would just get better.

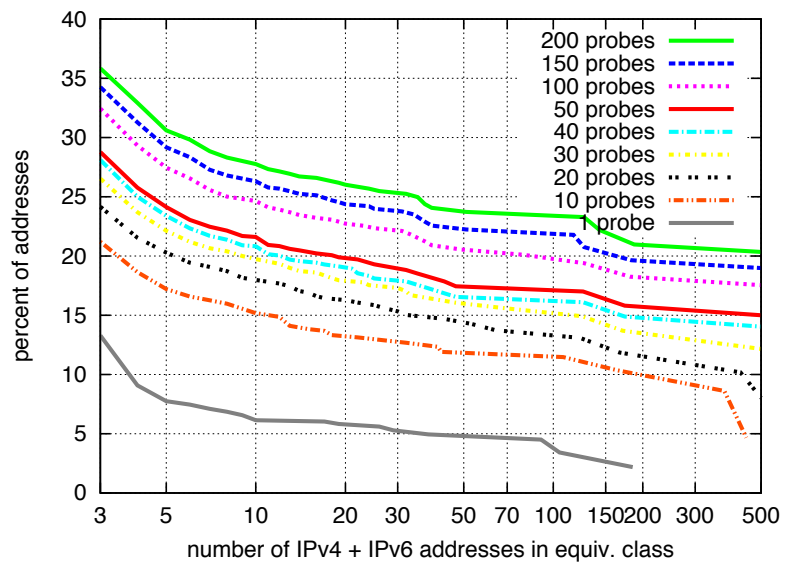

Figure 8: Percent of IPv4 + IPv6 addresses in equivalence classes of at least a given size, for open resolvers probed at least 200 times on April 19-20, 2013

\subsubsection{IPv4 address within IPv6 address (non-6to4)}

We mention briefly that of the few, 92, pairs with Teredo IPv6 addresses, (2001:0000: :/32) only one has an embedded IPv4 equal to the paired IPv4. Of the pairs where the IPv6 address is neither 6 to 4 nor Teredo, just $0.6 \%$ have an embedded IPv4 equal to the paired IPv4. Of these, $57 \%$ embed the IPv4 address in the lowest 32 bits.

We also confirm an interesting human-centric convention in at least one major ISP's DNS infrastructure that has been previously observed [16]. The operator assigns the lower 64 bits of the IPv6 address, using 16 bits to encode each octet of the IPv4 address, so that the hexadecimal values render as the decimal equivalent of the IPv4 address, such as $2001: 558: 1014: f: 68: 87: 76: 181$. which is paired with the IPv4 address 68.87.76.181.

\subsection{Discovery of Complexity in Resolver As- sociations Using Active DNS Measurements}

Here, we corroborate the passively discovered complexity using the Active DNS Measurement technique of $\S 2.2$ to perform a controlled data collection over a short time period. We first determine the open resolvers in the 6-month data set, for which we find about 7,000: 5,308 IPv4's and 1,677 IPv6's ${ }^{7}$. We probe each of these open resolvers via our active technique at least 200 times on April 19-20, 2013, requesting resolution for our special domain which induces chains of lookups across IPv6 and IPv4 glue. Thus, we obtain a data set over a short period of time, about 13 hours, and for which the same set of nameservers is probed repeatedly. 'Each 4-tuple of IPv6/IPv4/IPv6/IPv4 yields either 1, 2, or 4 (IPv4, IPv6) address pairs, depending on whether the two IPv4's and two v6's are the same.

We are interested in the marginal benefit of repeated active probing of the open resolvers, from the perspective of discovering the complexity in the IPv4, IPv6 nameserver associations using a different methodology than the passive

\footnotetext{
${ }^{7}$ While there exists a much larger population of open DNS resolvers in the Internet, we focus only on the subset of open resolvers that support IPv6 and appear within our passively collected six-month data set.
} 
technique. We examine those resolvers for which the 4-tuple is obtained at least 200 times, and then compute equivalence classes conditioned on different amounts of probing.

Figure 8 shows the percent of addresses that are in equivalence classes of at least a given size, as a function of the number of probes. The curves that are higher up in the Figure indicate that, overall, the equivalence classes are larger and more complex - the distribution has a heavier tail. The lowest curve in the plot is of equivalence classes of addresses from those 4-tuples discovered as a result of just the first probe to each of the resolvers. That is, even though at least 200 probes were made, the lowest curve considers just the first one. The next curve above that one is from those 4tuples discovered from the first ten probes, and so on. The Figure shows that with increased sampling, additional nameserver addresses are discovered, and the equivalence classes overall become larger. Note that the curves are stacked on top of each other, and where the spacing varies. There is a relatively large jump between the curve for 1 probe to that of 10 probes. Then, as progressively ten more probes are incorporated, up to 50 probes, the spacing tends to get smaller. And likewise, there is a relatively large jump between the curve for 1 probe to that of 50 probes, as compared with the incremental spacing from 50 to 100 , which in turn is larger than from 100 to 150 probes, and which is about the same from 150 to 200 probes. Thus, with the additional probing, additional existing complexity is discovered, though often the marginal increase is decreasing. Moreover (and particularly given the impact of going from 150 to 200 probes being about the same as from 100 to 150 ), even after 200 probes, in all likelihood we have not discovered all of the existing complexity associated with this given set resolvers. As with the six-month data set, the largest eq. classes have addresses that span multiple continents and many AS's.

We obtain analogous results to Figure 8 when in each eq. class, we aggregate the IPv6's to /64 prefixes. In summary, the active DNS measurement technique also finds complex associations, even when the probing is done over a short period of time. Repeated probing reveals additional complexity, though the marginal increase will decrease.

\subsection{Worthwhile to Record the Frequency Ad- dress Pairs are Observed}

Akamai's initial code that aggregated the logs of the Passive DNS Measurement technique did not compute a measure of the frequency that an address pair was observed. Jointly with the present study, Akamai is revising the code to include this measure.

In the present section we present one application of this measure: detecting the additional complexity in nameserver associations due to address pairs that are less frequently observed.

The 12-day data set is a one-time collection from Akamai's production deployment, and in contrast to the 6-month data set, includes the number of times an address pair was observed during this period. This data set is a precursor to the production deployment. The counts range from 1 to over 17 million, with a median value of 12 . The pairs with highest counts belong to Comcast and ATT.

We are interested in the impact of restricting the set of address pairs to those that were observed at least 12 times in the 12 days, as well as further restrictions. We start with the full data set, and remove pairs that contain the addresses
Table 4: The 1-1 and the largest equivalence class in the 12-day dataset, and subsets there of

\begin{tabular}{|c|c|c|c|c|}
\hline \multirow[b]{3}{*}{ Case } & $\%$ of & \multicolumn{3}{|c|}{ within the largest eq cl } \\
\hline & $\mathrm{IPv} 4+\mathrm{IP}$ & & & \\
\hline & $\begin{array}{l}\text { in } 1-1 \\
\text { eq cls }\end{array}$ & $\begin{array}{l}\text { Num of } \\
\text { IPv4 }\end{array}$ & $\begin{array}{l}\text { Num of } \\
\text { IPv6 }\end{array}$ & $\begin{array}{l}\text { Num of } \\
\text { pairs }\end{array}$ \\
\hline 12-day data set & $66 \%$ & 683 & 624 & 62,000 \\
\hline $\begin{array}{l}\text { at least } 12 \text { occur- } \\
\text { rences }\end{array}$ & $78 \%$ & 447 & 455 & 30,000 \\
\hline $\begin{array}{l}\text { omit AS } 15169 \& \\
6 \text { to4 }\end{array}$ & $72 \%$ & 346 & 47 & 764 \\
\hline $\begin{array}{l}\text { at least } 12 \text { occur- } \\
\text { rences; and omit } \\
\text { AS } 15169 \text { \& 6to } 4\end{array}$ & $82 \%$ & 1 & 34 & 34 \\
\hline $\begin{array}{l}\text { random subsets } \\
\text { from full data } \\
\text { set; same num of } \\
\text { pairs as prior line }\end{array}$ & $\begin{array}{l}76 \% \pm \\
0.7 \%\end{array}$ & $\begin{array}{ll}468 & \pm \\
5.7 & \end{array}$ & $\begin{array}{ll}508 & \pm \\
12.5\end{array}$ & $\begin{array}{l}11,112 \\
\pm 75.3\end{array}$ \\
\hline
\end{tabular}

in question, and then with the reduced set of pairs, we recompute the eq. classes. The results are shown in Table 4. The Table is comparable to Table 3 for the 6 -month data set, and also contains aspects of Table 2 .

As a sanity check, Table 4 includes info on the 1-1 eq. class. As shown in the first row, $66 \%$ of the addresses are in the 1-1 eq. class. This is in between the $34 \%$ for the 6 -month data set, and the $71 \%$ when that data set is restricted to the final week, where the latter is more comparable in duration to present case of 12 days.

The largest eq. class contains $52 \%$ of the address pairs, which is even a greater share than the $37 \%$ of the "mammoth" eq. class of the 6-month dataset (§4.1.3). When we omit the pairs whose addresses are in AS 15169 and whose IPv6 is 6to4, the third row, the number of pairs in the largest eq. classes is just 764 , which is about $1 \%$ of what it is for the full 12-day data set, the first row. This is the same percentage reduction as occurred with the 6 -month data set, Table 3. Now, when in addition we omit pairs with fewer that 12 occurrences, the number of pairs in the largest eq. class is just 34 , a further 20 fold reduction.

To distinguish from reduction due simply to a smaller number of pairs, we take ten random subsets of the full $12-$ day data set, where here the subsets have the same number of pairs as the fourth row of the Table 4, 21,000 pairs. The fifth row reports the mean value and range. The number of pairs in the largest eq. class is back up at 11,000, thus showing that the major cause for the relatively low complexity reported in line four is not due the smaller sample, but rather the selected pairs that were omitted.

\subsection{Validation}

To assess the IPv4 to IPv6 associations inferred via the passive DNS technique, we examine two sources of external information about the resolvers: PTR records and software version. Also, we were able to get feedback from three ISP's regarding sample data.

\subsubsection{PTR Records}

DNS PTR records map an IPv4 or IPv6 address to a human readable name. We perform a DNS PTR query for every address in the 6 -month dataset. For $14.5 \%$ of the pairs, both addresses have a PTR record. For these 97,821 pairs, Table 5 shows the percent of PTR record matches for the full data set, and then partitioned by those address pairs 
Table 5: Agreement between associated IPv4 and IPv6 equivalence classes and their corresponding DNS PTR records.

\begin{tabular}{|l|l|l|l|}
\hline Set & $\begin{array}{l}\text { \# Pairs } \\
\text { with PTR }\end{array}$ & $\begin{array}{l}\text { Exact } \\
\text { Match }\end{array}$ & $\begin{array}{l}\text { Second-Level } \\
\text { Match }\end{array}$ \\
\hline All & 97,821 & $4821(4.9 \%)$ & $47,752(48.8 \%)$ \\
\hline 1 -to-1 & 8461 & $3500(41.4 \%)$ & $5679(67.1 \%)$ \\
\hline Non 1-to-1 & 89,360 & $1321(1.5 \%)$ & $42,073(47.1 \%)$ \\
\hline
\end{tabular}

that are a 1-1 eq. class, and those in a larger eq. class. Of the addresses in 1-1 eq. classes, we see that $41 \%$ have PTR records that match exactly, as compared to only $1.5 \%$ of the non 1-1 eq. classes. Identical PTR records is supporting, though not definitive, evidence that the two addresses are either on the same server, or are the public-facing interfaces to a DNS server cluster.

Next, we examine the agreement between the IPv4 and IPv6 PTR records for just the second-level domain portion of the name, e.g. foo.bar.com and faz.bar.com match at the second-level. Whereas an exact PTR record match might suggest that the IPv4 and IPv6 address correspond to a common host, a load-balanced or distributed resolver might assign different hostnames to different machines, albeit with the same second-level domain. Supporting this conjecture is that while only $1.5 \%$ of the non $1-1$ eq. class pairs have exactly matching PTR records, $47.1 \%$ have a second-level PTR match. Whereas the difference is not as pronounced with pairs in $1-1$ eq. classes, with $41.4 \%$ matching exactly and $67.1 \%$ matching at the second-level. While DNS PTR records are frequently incorrect or misleading, these findings increase our confidence that our passive technique is discovering meaningful structure.

\subsubsection{Software Version}

We examine the version of software reported by the DNS resolvers discovered in the eq. classes of the 6-month dataset. To ascertain the software version, we perform a DNS Chaosnet class (CHAOS) TXT type query for the object version. bind. 35,921 of the addresses respond. An example response is:

"9.7.0-P2-RedHat-9.7.0-10.P2.e15_8.1"

For a baseline of what is the chance that two unrelated addresses have the same version.bind, we repeatedly randomly pick a IPv4 and a IPv6 address from this set (ignoring whether or not they are observed as a pair), which yields a $3 \%$ match rate.

For 8,475 of the address pairs, both addresses return a value for version.bind. As shown in Table 6 , the version matches for $82 \%$ of these pairs, a value significantly higher than the $3 \%$ random baseline. Moreover, $99.3 \%$ of the 1 1 eq. classes have resolvers that report exactly the same version.

Together, these PTR and version.bind results help support the validity of our associations and equivalence classes. While we can not definitively ascertain whether the two addresses of a pair are on the same machine, our in-progress work [8] considers other means to make a more definitive determination.

\subsubsection{Feedback from Network Operators}

We sent sample address associations to personal contacts at six major networks and got responses from three. All three confirmed that the IPv4 and IPv6 addresses of all of
Table 6: Agreement between associated IPv4 and IPv6 equivalence classes and their corresponding bind.version.

\begin{tabular}{|l|l|l|}
\hline Set & $\begin{array}{l}\text { Num of Pairs with } \\
\text { bind.version }\end{array}$ & $\begin{array}{l}\text { Exact } \\
\text { Match }\end{array}$ \\
\hline All & 8475 & $6951(82.0 \%)$ \\
\hline One-to-One & 4650 & $4619(99.3 \%)$ \\
\hline Non One-to-One & 3825 & $2332(61.0 \%)$ \\
\hline
\end{tabular}

the one-to-one associations that they checked are assigned to the same physical machine. One respondent, a cable operator, further explained that some of the addresses were of clients. Therefore, some of the associations we observe correspond to resolvers that are run on machines of customers of said cable operator.

\section{DISCUSSION}

This Section discusses additional details and implications of our methodology and results. In particular, we discuss the need to filter when making collocation inferences, and potential root causes for the non 1-1 eq. classes we observe.

\subsection{Screening for collocated addresses}

An interest in this paper is the discovery of IPv4, IPv6 address of resolvers that likely are on the same machine, or, from the viewpoint of geolocation, exist at a common location, e.g. data center. With this aim, we first wish to discover the complexity that exists in the Internet of IPv4, IPv6 resolver associations, so as to avoid the false conclusion of a simple association when reality is more complicated. Our findings demonstrate that, given the complexity of address associations, the observed pairs must be filtered in order to be most valuable to higher-level objectives, e.g. geolocation.

Natural candidates of value are address pairs that are in the 1-1 eq. class and have remained so over a period of time, such as six-months, or remained so after repeated, active probing, or repeated, passive observations. However, eq. classes larger than 1-1 also provide value, for instance eq. classes where the IPv6's differ only in the lower 64 bits, given the common practice that such addresses reside on a common subnet, or may be associated with a single interface where the interface ID is varied for anonymity.

Excluding non-native IPv6 addresses is natural as these only associate the IPv4 address with the infrastructure of a particular transition strategy. If we exclude address pairs whose IPv6 is 6to4, and then compute eq. classes, and aggregate IPv6's in the eq. classes to /64 prefixes, then for the six-month data set, we find that $50 \%$ of the addresses are associated with the 1-1 eq. classes of prefixes, which again is an appreciable proportion.

Having discovered address pairs that may be on the same server, we would like a technique that could make a definitive determination. In on-going work, we are developing an active, fingerprinting technique with that goal - see [8] for an initial description.

\subsection{Large Equivalence Classes}

There are several potential causes of equivalence classes larger than 1-1; for DNS resolvers specifically:

- DNS queries that subvert our multi-level hierarchy, e.g. manual or automated probes from different locations directed to specific nameservers. 
- Shared distributed caches, such as those commonly employed by large public DNS resolvers [15]. If NS records returned from the first-level are saved in a shared cache (with TTL of 12 hours for the present data set) and subsequently used by another resolver that sends a query over IPv6, then the second-level will discover multiple IPv6's associated with a single IPv4. A short TTL on the final A or AAAA record (20 seconds in the data set) increases reuse of the cached NS record. Furthermore, after the NS TTL expires and some other resolver using the shared cache does the lookup at the first-level, then another IPv4 address could be added to the equivalence class.

However, our results offer a more general caution in efforts that attempt to pair IPv4 and IPv6 addresses. Complicating factors that contribute to the complexity observed in our data, but are not specific to DNS and may affect other measurement efforts, include:

- Hosts and interfaces with multiple IPv4 and/or IPv6 addresses.

- Network Address Translation (NAT) [29] of either IPv4 or IPv6, where we observe multiple addresses of the non-translated protocol that correspond to a smaller set of addresses from the other. Such translation may occur at the edge, or within the carrier [20].

- Other forms of middleboxes, including load balancers, e.g. [3].

- Auto-tunneling, including 6to4, Teredo, etc [9].

- Address changes over time, including ephemeral addresses [31] and IPv6 privacy extensions [24].

\section{CONCLUSIONS}

This paper seeks to characterize the inter-relation of IPv4 and IPv6 among Internet DNS resolvers. We deploy both active and passive measurement techniques to discover and analyze sets of associated pairs of $\mathrm{IPv} 4, \operatorname{IPv} 6$ addresses.

While prior work has examined IPv4, IPv6 associations for clients, to our knowledge this paper is the first to begin to examine such associations for equipment within operators' networks, which can be a leading indicator of IPv6 adoption $[12,1]$.

We develop and deploy two novel measurement systems: i) a passive, opportunistic technique using a two-level DNS hierarchy that encodes IPv4 addresses within IPv6 nameserver records; and ii) an active DNS probing system that induces a combination of IPv4 and IPv6 DNS resolver lookups in a single resolution operation.

For a six-month passive data collection, we find that $34 \%$ of the addresses have a one-to-one association, i.e. appear in no other address pair. This percentage increases to almost 50\% when the IPv6's are aggregated to /64's. We consider this a positive result for finding candidate address pairs that likely are on the same server or subnet, and are collocated. Supporting evidence is provided via matching PTR records and software versions, and confirmation from three major Network Operators of sample pairings. Companion, in-progress work seeks to develop a fingerprinting technique that would confirm whether an address pair is on the same server, [8].
In contrast to such simple associations, we also find complex, connected sets of address pairs that, at the extreme, span continents and hundreds of ASes, where third-party resolvers with distributed shared caches [15] play a major role. We also observe multiple instances of abnormal use of 6 to 4 tunnels, where a 6 to 4 address is paired with hundreds of different IPv4's that again span multiple continents, and where the $\mathrm{IPv}_{4}$ embedded in the 6 to 4 does not equal any of the paired IPv4's.

The active technique, which was designed, implemented, and deployed completely separately from the passive one, also discovers significant complexity on the address-pair associations. It did so in the context of a controlled experiment of repeated probing of a given set of open resolvers over a short period of time, 13 hours.

The passive technique has been implemented in Akamai Technologies' DNS infrastructure, where the collected pairs, after appropriate filtering, are being used to bootstrap geolocation of IPv6 addresses, and were helpful for revisions to request-routing software to handle IPv6.

\section{Acknowledgments}

Matthew Levine had the original idea for the opportunistic nameserver technique, while Steve Hill, James Kretchmar, and Brian Sniffen contributed to its design and implementation at Akamai Technologies. Patrick Gilmore provided insights into common practice of network operators, and via his contacts obtained feedback on sample address pairs. Our shepherd, John Heidemann, helped to review and properly frame the work. Thanks to the anonymous reviewers, Mark Allman, Billy Brinkmeyer, Aaron Hughes, and Geoffrey Xie for invaluable feedback on initial drafts of this work. This work supported in part by NSF grants CNS-1111445, CNS1111672, and CNS-1213157. This material represents the position of the authors and not necessarily that of the NSF, Akamai Technologies, or the U.S. Government. 


\section{REFERENCES}

[1] IPv6 Implementors Conference, 2010. https://sites. google.com/site/ipv6implementors/2010/agenda.

[2] Public DNS: Performance Benefits, 2013. https://developers.google.com/speed/ public-dns/docs/performance.

[3] J. Abley. A software approach to distributing requests for DNS service, 2004. http: //ftp.isc.org/isc/pubs/tn/isc-tn-2004-1.html.

[4] B. Ager, W. Mühlbauer, G. Smaragdakis, and S. Uhlig. Comparing DNS resolvers in the wild. In Proceedings of the 10th ACM IMC, pages 15-21, 2010.

[5] Akamai. Edgescape geolocation, 2012. http://www . akamai.com/html/technology/ products/edgescape.html.

[6] R. Arends, R. Austein, M. Larson, D. Massey, and S. Rose. DNS Security Introduction and Requirements. RFC 4033, Mar. 2005.

[7] R. Beverly, A. Berger, Y. Hyun, and k. claffy. Understanding the efficacy of deployed internet source address validation filtering. In Proceedings of the 9th ACM IMC, pages 356-369, 2009.

[8] R. Beverly, A. Berger, N. Weaver, and L. Campbell. Inferring Internet Server IPv4 and IPv6 Address Relationships. Technical Report NPS-CS-13-002, Naval Postgraduate School, 2013. http: //calhoun.nps.edu/public/handle/10945/34089.

[9] B. Carpenter and K. Moore. Connection of IPv6 Domains via IPv4 Clouds. RFC 3056, Feb. 2001.

[10] k. claffy. Tracking IPv6 evolution: data we have and data we need. SIGCOMM Comput. Commun. Rev., 41(3):43-48, July 2011.

[11] L. Colitti, S. H. Gunderson, E. Kline, and T. Refice. Evaluating IPv6 adoption in the internet. In Proceedings of the 11th international conference on Passive and active measurement, PAM'10, pages 141-150, 2010.

[12] Comcast. IPv6 Information Center, 2012. http://www . comcast6. net/.

[13] S. Deering and R. Hinden. Internet Protocol, Version 6 (IPv6) Specification. RFC 2460, Dec. 1998.

[14] R. Elz and R. Bush. Clarifications to the DNS Specification. RFC 2181, July 1997.

[15] Google. Public DNS, 2012. https: //developers.google.com/speed/public-dns/.

[16] M. Heuse. Recent advances in IPv6 insecurities. In Chaos Communications Congress, 2010.
[17] G. Huston. IPv6 BGP Statistics, 2013. http://bgp.potaroo.net/v6/as2.0/.

[18] ISOC. World IPv6 Day, 2011. http://www . internetsociety.org/ipv6/ archive-2011-world-ipv6-day.

[19] ISOC. World IPv6 Launch, 2012. http://www . worldipv6launch.org.

[20] S. Jiang, D. Guo, and B. Carpenter. An Incremental Carrier-Grade NAT (CGN) for IPv6 Transition. RFC 6264 (Informational), June 2011.

[21] Z. M. Mao, C. D. Cranor, F. Douglis, M. Rabinovich, O. Spatscheck, and J. Wang. A Precise and Efficient Evaluation of the Proximity Between Web Clients and Their Local DNS Servers. In Proceedings of the General Track of the annual conference on USENIX Annual Technical Conference, ATEC '02, pages 229-242, 2002.

[22] P. Mockapetris. Domain names - implementation and specification. RFC 1035 (Standard), Nov. 1987.

[23] R. Mohan. Will U.S. Government Directives Spur IPv6 Adoption?, Sept. 2010. http://www.circleid.com/.

[24] T. Narten, R. Draves, and S. Krishnan. Privacy Extensions for Stateless Address Autoconfiguration in IPv6. RFC 4941 (Draft Standard), Sept. 2007.

[25] Nominum. Intelligent DNS, 2012. http://www. nominum. com/.

[26] E. Nygren, R. K. Sitaraman, and J. Sun. The Akamai network: a platform for high-performance internet applications. SIGOPS Oper. Syst. Rev., 44(3):2-19, Aug. 2010.

[27] RIPE NCC. World IPv6 Day Measurements, 2011. http://v6day.ripe.net.

[28] N. Sarrar, G. Maier, B. Ager, R. Sommer, and S. Uhlig. Investigating IPv6 traffic: what happened at the world IPv6 day? In Proceedings of PAM, 2012.

[29] P. Srisuresh and K. Egevang. Traditional IP Network Address Translator (Traditional NAT). RFC 3022 (Informational), Jan. 2001.

[30] P. Vixie. Extension Mechanisms for DNS (EDNS0). RFC 2671 (Proposed Standard), Aug. 1999.

[31] Y. Xie, F. Yu, K. Achan, E. Gillum, M. Goldszmidt, and T. Wobber. How dynamic are IP addresses? In Proceedings of SIGCOMM, pages 301-312, 2007.

[32] S. Zander, L. L. H. Andrew, G. Armitage, G. Huston, and G. Michaelson. Mitigating Sampling Error with Measuring Internet Client IPv6 Capabilities. In Proceedings of the SIGCOMM IMC, 2012. 